\title{
Baicalein pre-treatment alleviates hepatic ischemia/reperfusion injury in mice by regulating the Nrf2/ARE pathway
}

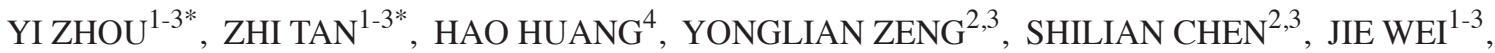 \\ GUOZHEN HUANG ${ }^{1-3}$, CHAOSI QIAN ${ }^{1-3}$, GUANDOU YUAN ${ }^{1-3}$ and SONGQING HE HE $^{1-3}$ \\ ${ }^{1}$ Division of Hepatobiliary Surgery, The First Affiliated Hospital of Guangxi Medical University; \\ ${ }^{2}$ Key Laboratory of Early Prevention and Treatment for Regional High Frequency Tumor (Guangxi Medical University), \\ Ministry of Education; ${ }^{3}$ Guangxi Key Laboratory of Early Prevention and Treatment for Regional High Frequency Tumor, \\ Department of Science and Technology of Guangxi, Nanning, Guangxi 530021; ${ }^{4}$ Division of Hepatobiliary \\ Surgery, Affiliated Hospital of Guilin Medical University, Guilin, Guangxi 541001, P.R. China
}

Received June 11, 2021; Accepted August 4, 2021

DOI: $10.3892 /$ etm.2021.10816

\begin{abstract}
Hepatic ischemia-reperfusion injury (HIRI) is caused by blood flow recovery following ischemia. Baicalein (BAI), a natural antioxidant used in traditional Chinese medicine, eliminates excessive free radicals and protects the structure of the cell membrane. However, its protective mechanism against HIRI is still unclear. The present study investigated underlying mechanism using a mouse HIRI model. Liver injury was evaluated using serum levels of alanine aminotransferase and aspartate aminotransferase, and hematoxylin-eosin staining was performed to evaluate the pathological changes in liver tissue. Apoptosis of hepatocytes was detected by TUNEL staining. The expression levels of reactive oxygen species (ROS), malondialdehyde (MDA) and superoxide dismutase (SOD) in the liver were detected to evaluate oxidative stress. Western blotting was performed to assess expression levels of nuclear factor E2-related factor 2 (Nrf2)/antioxidant response elements (ARE) pathway proteins in liver tissue. BAI pre-treatment significantly decreased elevation of serum aminotransferase levels induced by IR and alleviated histological damage to the liver. BAI decreased production of ROS and MDA in liver tissue induced by IR and increased the activity of SOD. At the same time, BAI inhibited apoptosis of liver cells induced by oxidative stress. Furthermore, BAI promoted the translocation of Nrf2 into the
\end{abstract}

Correspondence to: Professor Songqing He or Dr Guandou Yuan, Division of Hepatobiliary Surgery, The First Affiliated Hospital of Guangxi Medical University, 6 Shuangyong Road, Nanning, Guangxi 530021, P.R. China

E-mail: dr_hesongqing@163.com

E-mail: yuan510149375@163.com

*Contributed equally

Key words: baicalein, nuclear factor E2-related factor 2 signaling, reactive oxygen species, hepatic ischemia/reperfusion injury nucleus and increased the expression of total heme oxygenase-1 and NAD(P)H dehydrogenase quinone-1. The Nrf2 inhibitor ML385 reversed the protective effect of BAI on HIRI. These results indicated that BAI served a protective effect in HIRI by regulating the Nrf2/ARE pathway.

\section{Introduction}

Hepatic ischemia-reperfusion injury (HIRI) occurs in hepatectomy, severe liver trauma surgery and liver transplantation (1). It results in postoperative liver dysfunction and failure (2). Reactive oxygen species (ROS) are one of the key factors influencing HIRI $(3,4)$. ROS are continuously produced during hepatic ischemia-reperfusion and excessive levels ROS damage hepatocytes $(5,6)$. In response to ROS damage, liver cells undergo inflammation and apoptosis (7). The liver forms a complex oxidative stress response system and upregulates expression levels of protective genes, such as heme oxygenase-1 (HO-1), to decrease injury to hepatocytes when exposed to ROS (8). Previous studies have shown that pre-treatment drugs, such as sulforaphane and baicalein (BAI), can also regulate the antioxidant pathway in the liver $(9,10)$.

BAI is one of the most abundant flavonoids and the primary active component of Scutellaria baicalensis $(11,12)$. Previous studies have confirmed that BAI has anti-bacterial, ROS scavenging, anti-inflammatory and anti-tumor effects (13-16). Moreover, accumulated data have shown that BAI protects against several types of liver disease, such as alcoholic liver disease $(17)$, non-alcoholic fatty liver disease $(18,19)$, chemical-induced liver fibrosis (20) and immunological liver injury (21). Although certain studies by Liu et al $(22,23)$ addressed the beneficial effects of BAI on HIRI, no in vivo study has determined the mechanism underlying the protective effects of BAI on HIRI via the nuclear factor E2-related factor 2 (Nrf2)/antioxidant response element (ARE) pathway.

Nrf2 has been found to regulate antioxidant proteins by interacting with ARE, which is a key endogenous antioxidant stress pathway (9). There are $>200$ coding endogenous protective genes regulated by the Nrf2/ARE signaling pathway (1). These protective genes include antioxidant and phase II 
detoxification enzymes, such as catalase, HO-1, superoxide dismutase (SOD), and NAD $(\mathrm{P}) \mathrm{H}$ dehydrogenase quinone (NQO)-1 and -2 (24-26). According to previous studies, the Nrf2/ARE pathway serves a key role in protection against HIRI in mice $(8,27,28)$. However, whether the Nrf2/ARE pathway also contributes to the protection provided by BAI against HIRI remains unclear. The present study investigated the role of the Nrf2/ARE pathway in the protection effect provided by BAI against HIRI and its underlying mechanism.

\section{Materials and methods}

Animals. A total of 72 male C57BL/6 mice (age 6-8 weeks; body weight, 19-21 g) were obtained from the Animal Center of Guangxi Medical University (Nanning, China). Three mice were housed per individual standard cages under controlled conditions $\left(22 \pm 1{ }^{\circ} \mathrm{C}, 60 \pm 10 \%\right.$ relative humidity and $12 \mathrm{~h}$ light/dark cycles), with free access to water and food. BAI was obtained from Yuanye Bio (cat. no. S25956), with a $>98 \%$ purity (Fig. 1A). Nrf2 inhibitor ML385 (cat. no. SML1833) was obtained from Sigma-Aldrich (Merck KGaA). Mice were randomly assigned into different groups ( $n=6 /$ group) as follows: i) Sham, following intraperitoneal injection of DMSO, the abdominal cavity was opened and closed without IR; ii) BAI (100 mg/kg), following intraperitoneal injection of BAI (100 mg/kg; dissolved in DMSO), the abdominal cavity was opened and closed without IR; iii) IR, DMSO was injected intraperitoneally before IR; iv) BAI $(10 \mathrm{mg} / \mathrm{kg})+\mathrm{IR}$, BAI $(10 \mathrm{mg} / \mathrm{kg})$ was injected intraperitoneally before IR; v) BAI $(50 \mathrm{mg} / \mathrm{kg})+\mathrm{IR}$, BAI $(50 \mathrm{mg} / \mathrm{kg})$ was injected intraperitoneally before IR; vi) BAI (100 mg/kg) + IR, BAI (100 mg/kg) was injected intraperitoneally before IR; vii) ML385 (30 mg/kg) + IR, ML385 (30 mg/kg) was injected intraperitoneally before IR; and viii) ML385 (30 mg/kg) + BAI (100 mg/kg) + IR, ML385 (30 mg/ $\mathrm{kg})$ and BAI (100 mg/ $\mathrm{kg})$ were injected intraperitoneally before IR. The IR and BAI $(100 \mathrm{mg} / \mathrm{kg})+$ IR groups were further divided into reperfusion subgroups, with 6 mice per subgroup, (1, 6 and 24 h). Mice were euthanized at 1, 6 and $24 \mathrm{~h}$ post-HIRI. All animals were treated humanely and all animal experiments were approved by the Animal Experimental Ethics Committee of the First Affiliated Hospital of Guangxi Medical University (Nanning, China).

HIRI model. General anesthesia was induced in all mice by continuous spontaneous inhalation of 2-5\% volatile anesthetic isoflurane via a mask. A mouse model of HIRI was established as described by Tsung et al (29). The midline incision of the upper abdomen was performed to separate and expose the hepatic portal. The branches of the left and middle portal veins and hepatic arteries were clipped using a non-invasive vascular clamp, resulting in $70 \%$ warm ischemia. The color of the liver lobe in the blocked area changed from bright red to pale white, indicating successful induction of hepatic ischemia. After $60 \mathrm{~min}$, the clamp was released and blood flow was restored. In the Sham and BAI (100 mg/kg) groups, only the liver was exposed and blood flow was not blocked. After 1,6 and $24 \mathrm{~h}$ reperfusion, mice were anesthetized via inhalation of 3\% isoflurane for 3-5 min and 0.8-1 ml blood samples from the inferior vena cava and liver tissue were collected. Following blood collection, all mice were euthanized in a closed chamber via inhalation of 5\% volatile isoflurane for $10 \mathrm{~min}$. Mouse death was confirmed by cessation of heartbeat and nerve reflex. The procedure is shown in Fig. 1B.

Liver function assessment. Blood samples were stored at room temperature (RT) for $1 \mathrm{~h}$ and then were centrifuged at $1,413 \times \mathrm{g}, 4^{\circ} \mathrm{C}$ for $15 \mathrm{~min}$. Serum was collected and stored at $-80^{\circ} \mathrm{C}$ before testing. The levels of alanine aminotransferase (ALT) (cat. no. C001-e; Changchun Huili Biotech Co., Ltd.) and aspartate aminotransferase (AST) (cat. no. C002-e, Changchun Huili Biotech Co., Ltd.) were determined using a 7180 Biochemical Analyzer (Hitachi, Ltd.).

Histopathological observation and evaluation of liver injury. Ischemic liver lobes were fixed in $10 \%$ formalin at RT for $24 \mathrm{~h}$ and embedded in paraffin. Then, $5 \mu \mathrm{m}$ thick paraffin slices were dewaxed with xylene twice (10 min each), fully hydrated with ethanol twice (5 min each) and rinsed with tap water. Sections were stained with hematoxylin solution (kit cat. no. C0105S; Beyotime Institute of Biotechnology) at RT for 3-5 min, rinsed with tap water and treated with $1 \%$ hematoxylin differentiation solution at RT for 6-25 sec. Sections were treated with $0.6 \%$ ammonia water solution for bluing at RT for $90 \mathrm{~min}$, rinsed with tap water and immersed in 85 and $95 \%$ ethanol at RT for 5 min each. Sections were stained with $0.5 \%$ eosin dye (kit cat. no. C0105S; Beyotime Institute of Biotechnology) at RT for $5 \mathrm{~min}$. The sections were then dehydrated and hyalinized in xylene before being sealed in neutral gum. Liver damage was determined under a light microscope (Olympus Corporation) (magnification, $\mathrm{x} 400$ ). The degree of liver injury was evaluated by two independent pathologists blinded to the experimental groups according to the classification standard described by Suzuki et al (30). This Suzuki grading standard consists of five grades (0-4) according to vacuolization of hepatocyte cytoplasm, necrosis and tissue congestion.

TUNEL detection of apoptosis. TUNEL was used to detect hepatocyte apoptosis (Colorimetric TUNEL Apoptosis Assay kit; cat. no. C1098; Beyotime Institute of Biotechnology) according to the manufacturer's protocol. Ischemic liver lobes were fixed in $10 \%$ formalin at RT for $24 \mathrm{~h}$ and embedded in paraffin. The $5 \mu \mathrm{m}$ thick paraffin slices were dewaxed with xylene twice for $10 \mathrm{~min}$ each, hydrated with ethanol twice for 5 min each, followed by dehydration using 95, 90, 80 and 70\% ethanol ( 5 min each). Proteinase $\mathrm{K}$ working solution was added at $37^{\circ} \mathrm{C}$ for $20 \mathrm{~min}$. Sections were immersed in $3 \% \mathrm{H}_{2} \mathrm{O}_{2}$ at RT for $20 \mathrm{~min}$, then washed three times with PBS ( $\mathrm{pH} 7.4)$ in a Rocker device. A total of $50 \mu \mathrm{l}$ TUNEL reaction mixture was added at $37^{\circ} \mathrm{C}$ for $1 \mathrm{~h}$. Streptavidin-horseradish peroxidase and Tris-buffered saline containing 0.1\% (v/v) Tween-20 (TBST) were mixed at a ratio of 1:200, added to cover the tissue and incubated at $37^{\circ} \mathrm{C}$ for $30 \mathrm{~min}$. The slides were placed in PBS (pH 7.4) and washed with shaking on a decolorizing shaker three times ( $5 \mathrm{~min}$ each). Then, diaminobenzidine coloring solution $(0.2 \mathrm{ml})$ provided in the kit was added to marked tissue at RT for $5 \mathrm{~min}$. Sections were counterstained with hematoxylin staining solution at RT for $1 \mathrm{~min}$ and washed in water, dried and sealed with neutral resin (cat. no. G8590; Beijing Solarbio Science \& Technology Co., Ltd.). TUNEL-positive 
A

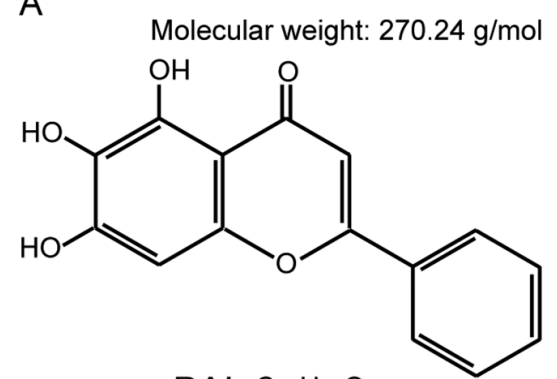

BAI: $\mathrm{C}_{15} \mathrm{H}_{100}$
B

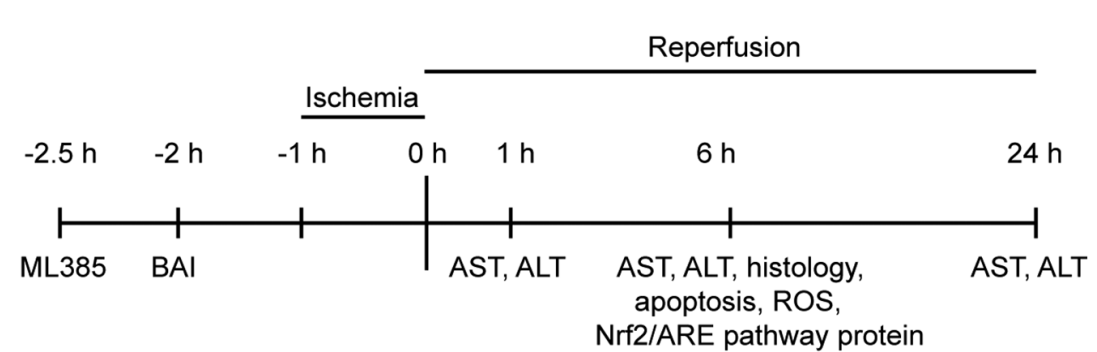

Figure 1. Structure of BAI and schematic diagram of the experimental protocol. (A) Chemical structure of BAI. (B) Experimental model design. BAI, baicalein; AST, aspartate aminotransferase; ALT, alanine aminotransferase; ROS, reactive oxygen species; Nrf2, nuclear factor E2-related factor 2; ARE, antioxidant response elements; IR, ischemia/reperfusion.

hepatocytes (brown-yellow nucleus) were counted in $>3$ high power histological fields under a light microscope (Olympus Corporation; magnification, $\mathrm{x} 400$ ) and the percentage was calculated.

Oxidative stress evaluation. Ischemic liver tissue was perfused with normal saline three times at $4^{\circ} \mathrm{C}$ for $3 \mathrm{~min}$ and ground into $10 \%$ homogenate. The homogenate was centrifuged at $1,413 \mathrm{x} \mathrm{g}$ and $4^{\circ} \mathrm{C}$ for $10 \mathrm{~min}$. The total protein concentration of the supernatant was determined via BCA method. The content of SOD (cat. no. S0103; Beyotime Institute of Biotechnology) and malondialdehyde (MDA; cat. no. S0131S; Beyotime Institute of Biotechnology) in liver tissue homogenate from each group was determined according to the manufacturer's instructions. Frozen sections $(10 \mu \mathrm{m})$ were prepared from fresh liver tissue and the ROS content was determined by a fluorescent dye dihydroethidium (DHE) (cat. no. D7008; Sigma-Aldrich; Merck KGaA). Tissue at RT was marked liquid blocker pen. Spontaneous fluorescence quenching reagent was added at RT for 5 min before washing in running tap water for $10 \mathrm{~min}$. ROS staining solution (DHE $10 \mu \mathrm{mol} / \mathrm{l}$ ) was added to the tissue area at $37^{\circ} \mathrm{C}$ for $30 \mathrm{~min}$ in the dark. Sections were washed three times with PBS ( $\mathrm{pH} 7.4)$ in a Rocker device (5 min each), incubated with DAPI solution (cat. no. G1012; Wuhan Servicebio Technology Co., Ltd.) at RT for 10 min in the dark and washed three times with PBS (pH 7.4) in a Rocker device (5 min each). Samples were mounted with anti-fade mounting medium and images were captured using a fluorescence microscope (magnification, x400). DAPI glows blue under UV at excitation wavelength 330-380 nm and emission wavelength $420 \mathrm{~nm}$; DHE itself displays blue fluorescence (absorption/emission, 355/420 nm) in cell cytoplasm while the oxidized form ethidium displays red fluorescence (absorption/emission, 510/590 nm) upon DNA intercalation. Nuclei appear blue by labeling with DAPI. ROS-positive cells were labelled by fluorescein are red.

Western blotting. Total protein of HO-1 and NQO-1 from each group was extracted using RIPA lysis buffer (cat. no. R0010; Beijing Solarbio Science \& Technology Co., Ltd.). The level of Nrf2 nucleoprotein was measured in nuclear lysate by a nuclear protein extraction kit (cat. no. R0050; Beijing Solarbio Science \& Technology Co., Ltd.). The protein concentration was determined using a BCA kit (cat. no. P0010S; Beyotime
Institute of Biotechnology). A total of $20 \mu \mathrm{g} /$ lane protein samples were separated using 10\% SDS-PAGE and transferred to PVDF membranes (cat. no. IPVH00010; Merck KGaA). Membranes were blocked with 5\% skimmed milk at RT for $1 \mathrm{~h}$. The membranes were incubated with primary antibodies at $4^{\circ} \mathrm{C}$ overnight. The primary antibodies were as follows: Anti-Nrf2 (1:1,000; cat. no. T55136; Abmart Pharmaceutical Technology Co., Ltd.), anti-HO-1 (1:1,000; cat. no. PY5393; Abmart Pharmaceutical Technology Co., Ltd.), anti-NQO-1 (1:1,000; cat. no. T56710; Abmart Pharmaceutical Technology Co., Ltd.), anti-Histone H3 (1:1,000; cat. no. T56587; Abmart Pharmaceutical Technology Co., Ltd.) and anti-GAPDH (1:20,000; cat. no. 10494-1-AP; ProteinTech Group, Inc.). Membranes were then washed three times in Tris-buffered saline containing $0.1 \%$ (v/v) Tween-20 (TBST) and incubated with horseradish-conjugated goat-anti-rabbit secondary antibody (1:10,000; cat. no. SA00001-2; ProteinTech Group, Inc.) at RT for $1 \mathrm{~h}$. Membranes were washed again. ECL reagent (cat. no. P0018FM, Beyotime Institute of Biotechnology) was used to visualize the bands. Bands were detected with a GeneSys System (Bio Rad Laboratories, Inc.). Band intensities were measured using Image J v1.8.0 software (National Institutes of Health).

Statistical analysis. SPSS 13.0 (SPSS, Inc.) and GraphPad Prism 5.0 software (GraphPad Software, Inc.) were used for statistical analysis and presentation of the data. Shapiro-Wilk test confirmed that data were normally distributed. The data are presented as mean \pm SD of at least three replicates. One- or two-way ANOVA followed post hoc Tukey's or Holm-Sidak test, respectively, were performed for multiple group comparisons. Two-sided $\mathrm{P}<0.05$ was considered to indicate a statistically significant difference.

\section{Results}

BAI ameliorates liver injury induced by IR. The activity of ALT and AST in serum and histological examination were used to assess liver injury. Levels of AST and ALT in the IR group were significantly increased, indicating impaired liver function (Fig. 2A and B). Serum ALT and AST levels were significantly decreased by pre-treatment with medium- and high-dose BAI (50 and $100 \mathrm{mg} / \mathrm{kg}$ ) before IR, which indicated that BAI exerted a significant protective effect on liver func- 

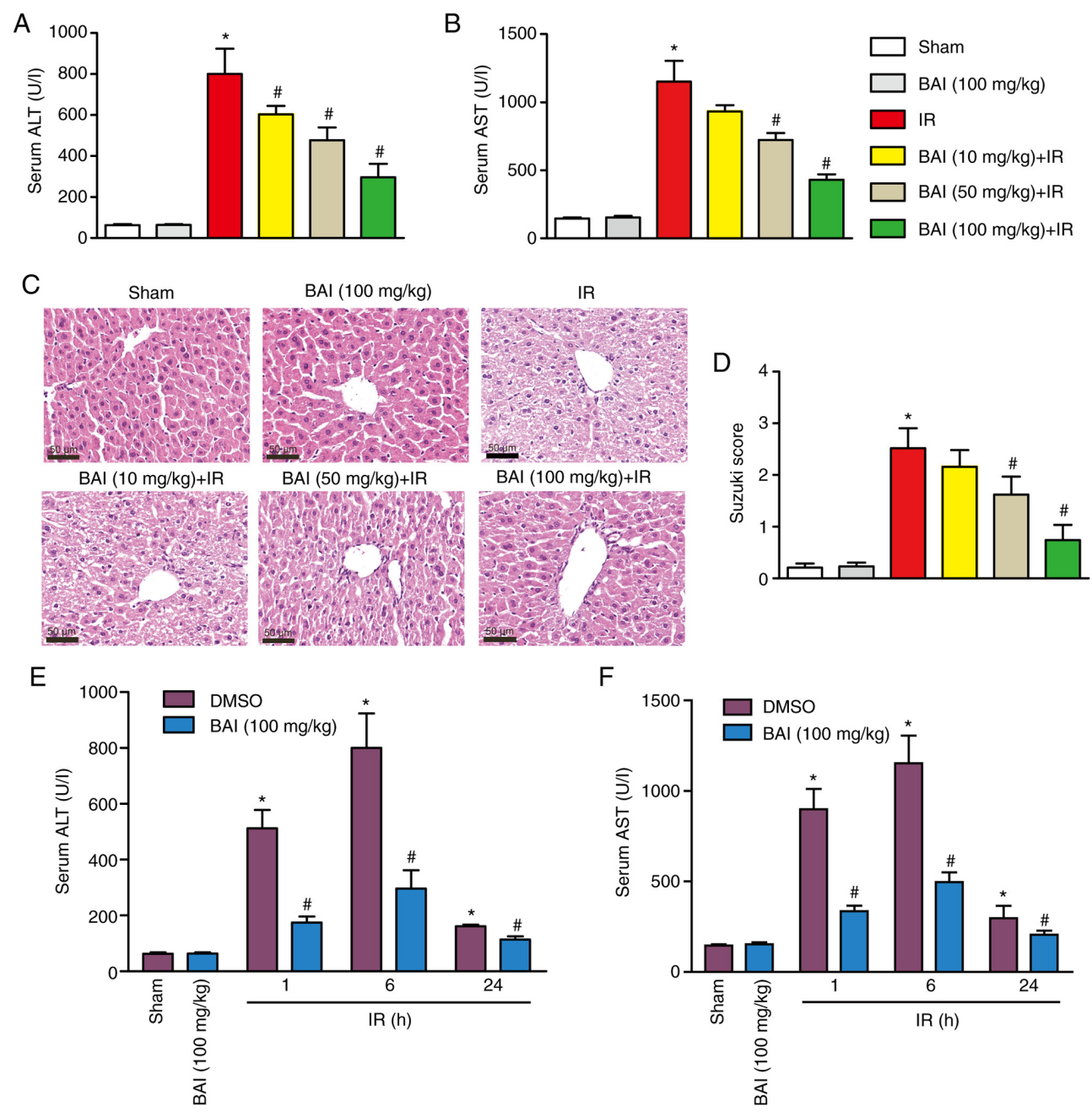

Figure 2. BAI ameliorates liver damage induced by IR. Mice were pre-treated with either vehicle (DMSO) or BAI at $10,50 \mathrm{or} 100 \mathrm{mg} / \mathrm{kg}$. Serum levels of (A) ALT and (B) AST were assessed and (C) Hematoxylin and eosin staining was performed $6 \mathrm{~h}$ after hepatic IR. Scale bar $=50 \mu \mathrm{m}$. (D) Suzuki score. ${ }^{*} \mathrm{P}<0.05 \mathrm{vs}$. Sham; ${ }^{*} \mathrm{P}<0.05$ vs. IR. The effect of BAI on serum (E) ALT and (F) AST levels in mice subjected to Sham or ischemia treatment followed by reperfusion. ${ }^{*} \mathrm{P}<0.05$ vs. Sham; ${ }^{*} \mathrm{P}<0.05$ vs. DMSO at the same time point. BAI, baicalein; AST, aspartate aminotransferase; ALT, alanine aminotransferase; IR, ischemia/reperfusion.

tion. Consistent with the alterations in serum ALT and AST levels, compared with the Sham and BAI $(100 \mathrm{mg} / \mathrm{kg})$ group, a larger necrotic area was observed in the IR group by HE staining (Fig. 2C). Compared with the I/R group, pre-treatment with medium- and high-dose BAI $(50$ and $100 \mathrm{mg} / \mathrm{kg}$ ) before IR significantly decreased liver necrosis. Pathological results were verified by Suzuki score (Fig. 2D). Suzuki scores indicated that the average injury level in the I/R group was significantly higher than that in the Sham and BAI $(100 \mathrm{mg} / \mathrm{kg})$ groups. However, compared with the I/R group, the BAI $(50 \mathrm{mg} / \mathrm{kg})+$ IR and BAI $(100 \mathrm{mg} / \mathrm{kg})+$ IR groups showed significant alleviation of average injury levels, while there was no significant difference between injury levels in the $\mathrm{I} / \mathrm{R}$ and BAI $(10 \mathrm{mg} / \mathrm{kg})+\mathrm{IR}$ groups. Following reperfusion $(1,6$ and $24 \mathrm{~h})$, pre-treatment with BAI significantly decreased the levels of ALT and AST (Fig. 2E and F) compared with the corresponding IR group. These results suggested that BAI protected the liver from HIRI.

BAI decreases oxidative stress and hepatic apoptosis following HIRI. The aforementioned results indicated that medium- and high dose BAI (50 and $100 \mathrm{mg} / \mathrm{kg}$ ) had protec- tive effects on HIRI and $100 \mathrm{mg} / \mathrm{kg}$ had a better protective effect. The levels of ALT and AST peaked at $6 \mathrm{~h}$ after IR; therefore, this time point was selected for subsequent experiments. To determine the protective effect of $100 \mathrm{mg} / \mathrm{kg}$ BAI, both blood and liver tissue samples from the Sham, BAI $(100 \mathrm{mg} / \mathrm{kg})$, IR and BAI $(100 \mathrm{mg} / \mathrm{kg})+$ IR groups were used for further study. To assess the effect of BAI on oxidative damage to the liver, liver tissue was stained with DHE probe to detect liver ROS content. Compared with the Sham and BAI $(100 \mathrm{mg} / \mathrm{kg})$ groups, IR liver tissue samples showed notably higher expression levels of intracellular ROS (Fig. 3A). Pre-treatment with BAI suppressed ROS levels. Pre-treatment with BAI $(100 \mathrm{mg} / \mathrm{kg})$ before IR significantly decreased liver cell apoptosis compared with the IR group (Fig. 3B and C). Moreover, compared with the Sham and BAI $(100 \mathrm{mg} / \mathrm{kg})$ groups, the MDA content in liver tissue of the IR group was significantly increased; this was downregulated significantly by BAI (100 mg/kg) pre-treatment (Fig. 3D). By contrast to MDA, the activity of SOD in liver tissue was decreased significantly in the IR group compared with the Sham group (Fig. 3E). Pre-treatment with BAI increased the activity of SOD in liver tissue independently of IR. 
A
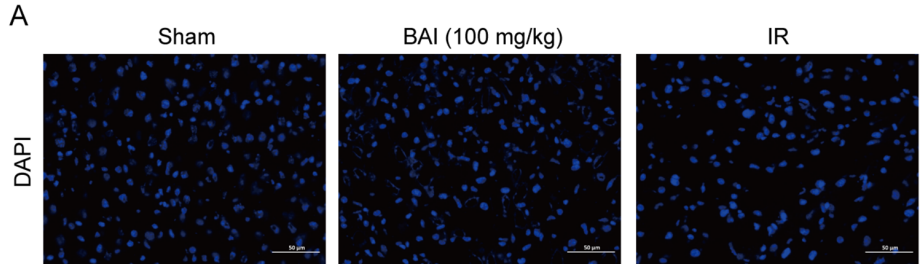

BAI $(100 \mathrm{mg} / \mathrm{kg})+\mathrm{IR}$
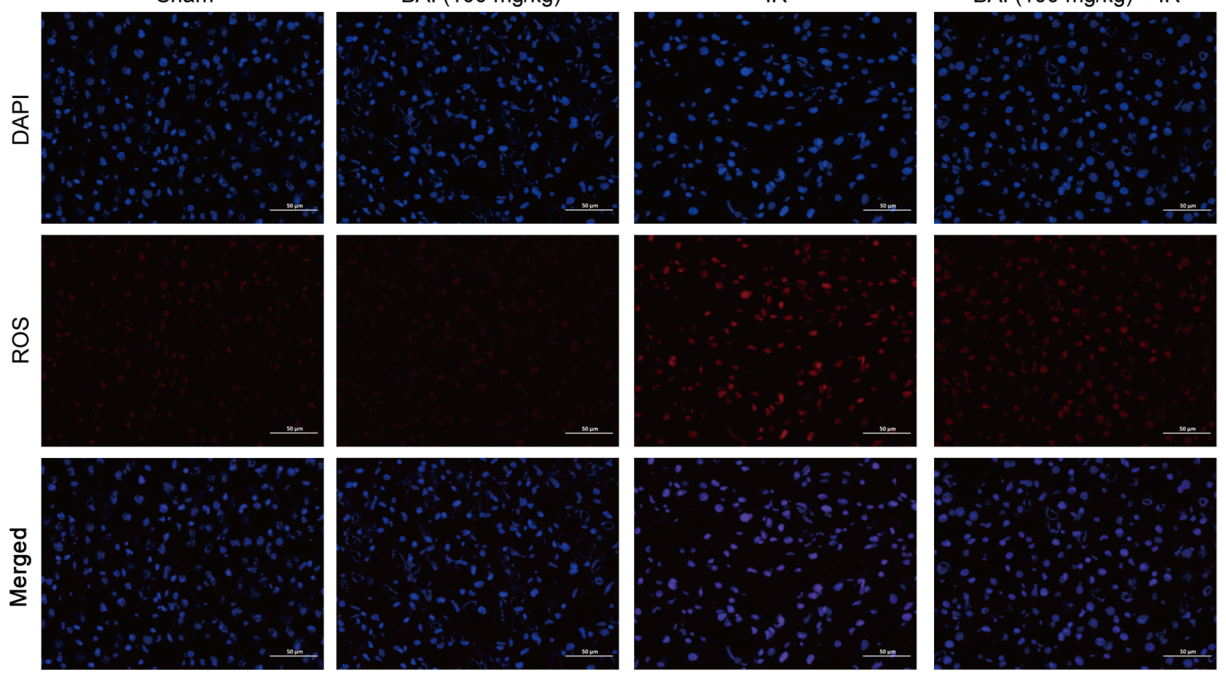

B

Sham

$\mathrm{BAl}(100 \mathrm{mg} / \mathrm{kg})$
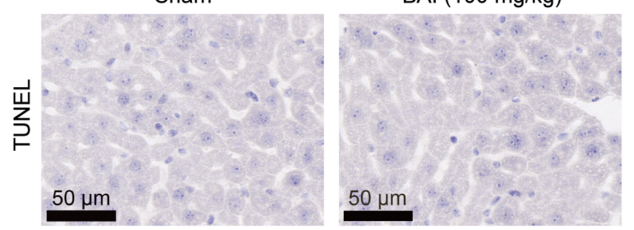

IR
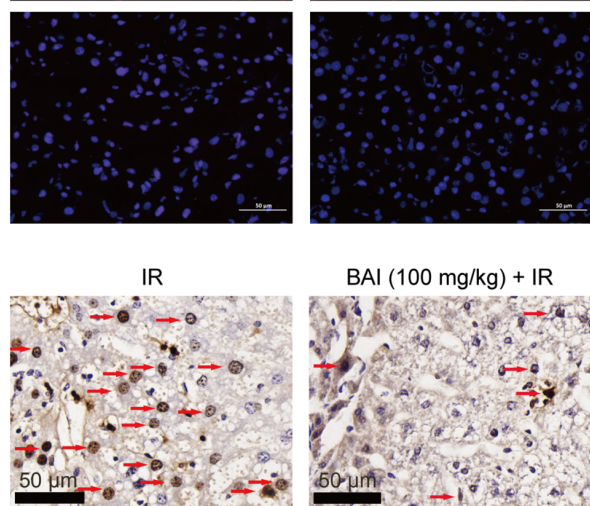

$\mathrm{BAI}(100 \mathrm{mg} / \mathrm{kg})+\mathrm{IR}$
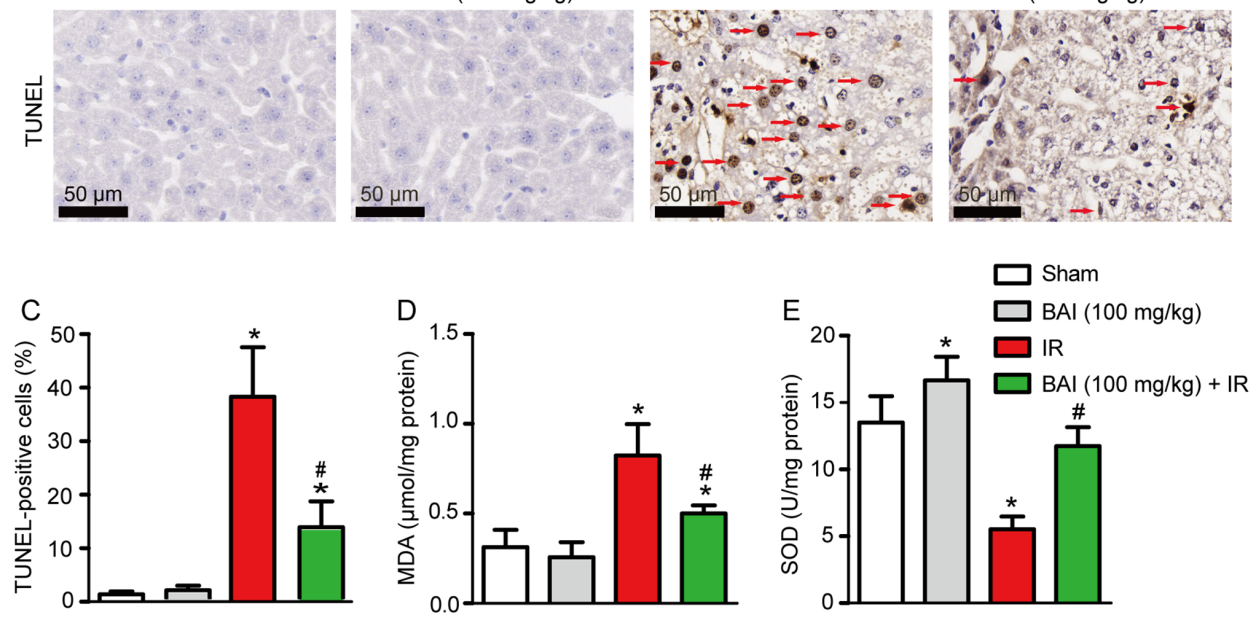

Figure 3. BAI decreases oxidative stress and hepatic apoptosis following I/R. Samples were collected $6 \mathrm{~h}$ after liver reperfusion in the I/R and BAI $(100 \mathrm{mg} / \mathrm{kg})+$ IR groups. (A) ROS (red) in liver tissue. (B) Representative images of TUNEL staining. Arrows indicate positive cells. Scale bar=50 $\mu \mathrm{m}$. (C) Percentage of TUNEL-positive hepatocytes. (D) MDA content and (E) SOD activity were measured in liver tissue. ${ }^{~ P}<0.05$ vs. Sham; ${ }^{*} \mathrm{P}<0.05$ vs. IR. BAI, baicalein; IR, ischemia/reperfusion; ROS, reactive oxygen species; MDA, malonaldehyde; SOD, superoxide dismutase.
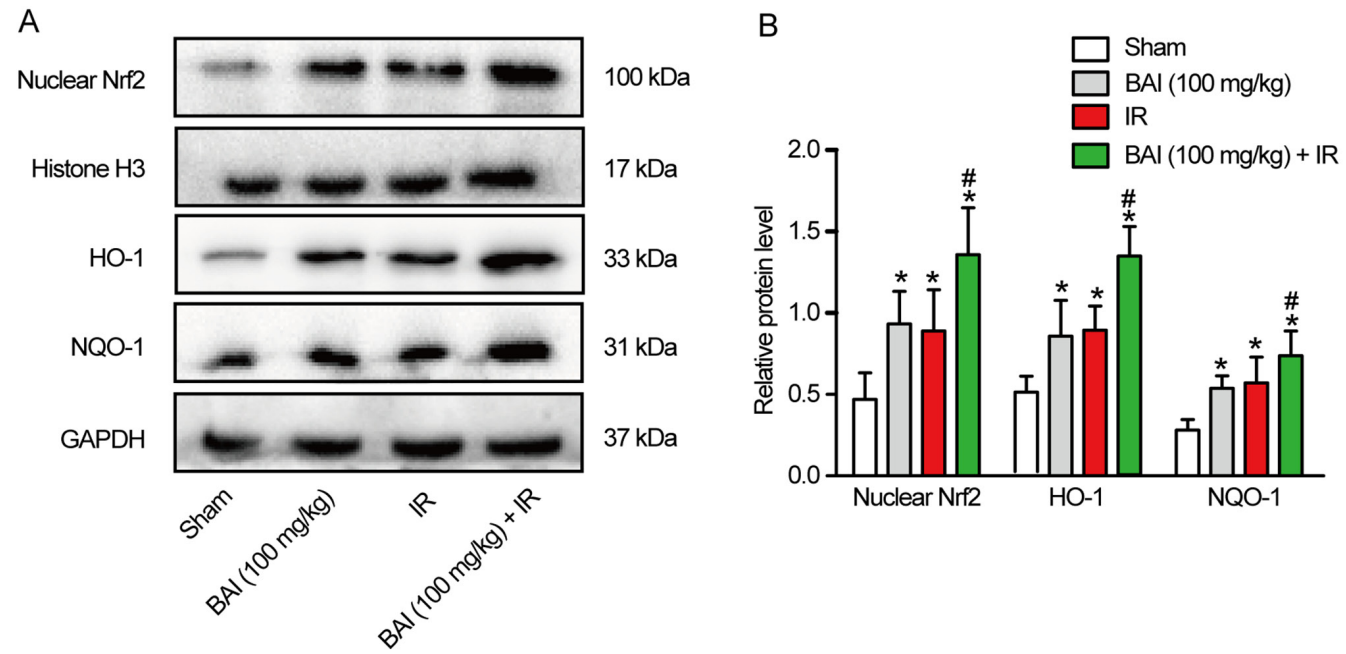

Figure 4. BAI upregulates Nrf2/ARE pathway protein expression in the liver. Samples were collected $6 \mathrm{~h}$ after liver reperfusion in the IR and BAI $(100 \mathrm{mg} / \mathrm{kg})+$ IR groups. (A) Levels of Nrf2 nucleoprotein, total HO-1 and NQO-1 protein were analyzed by western blotting. Histone H3 and GAPDH were used as the internal controls. (B) Nrf2 nucleoprotein relative to histone $\mathrm{H} 3$ and total HO-1 and NQO-1 protein relative to GAPDH. "P<0.05 vs. Sham. "P $<0.05$ vs. IR. BAI, baicalein; IR, ischemia/reperfusion; Nrf2, nuclear factor E2-related factor 2; ARE, antioxidant response elements; HO01, heme oxygenase-1; NQO-1, NAD $(\mathrm{P}) \mathrm{H}$ dehydrogenase quinone-1. 

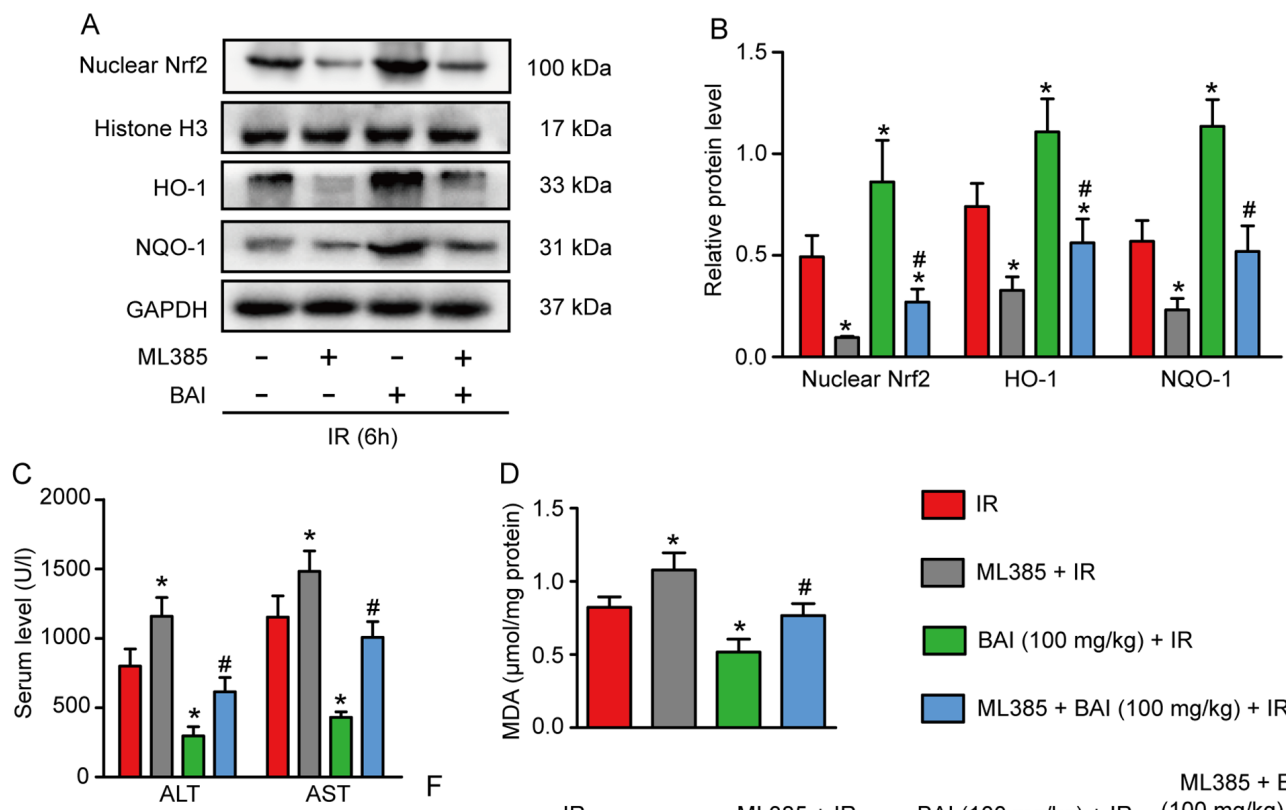

D
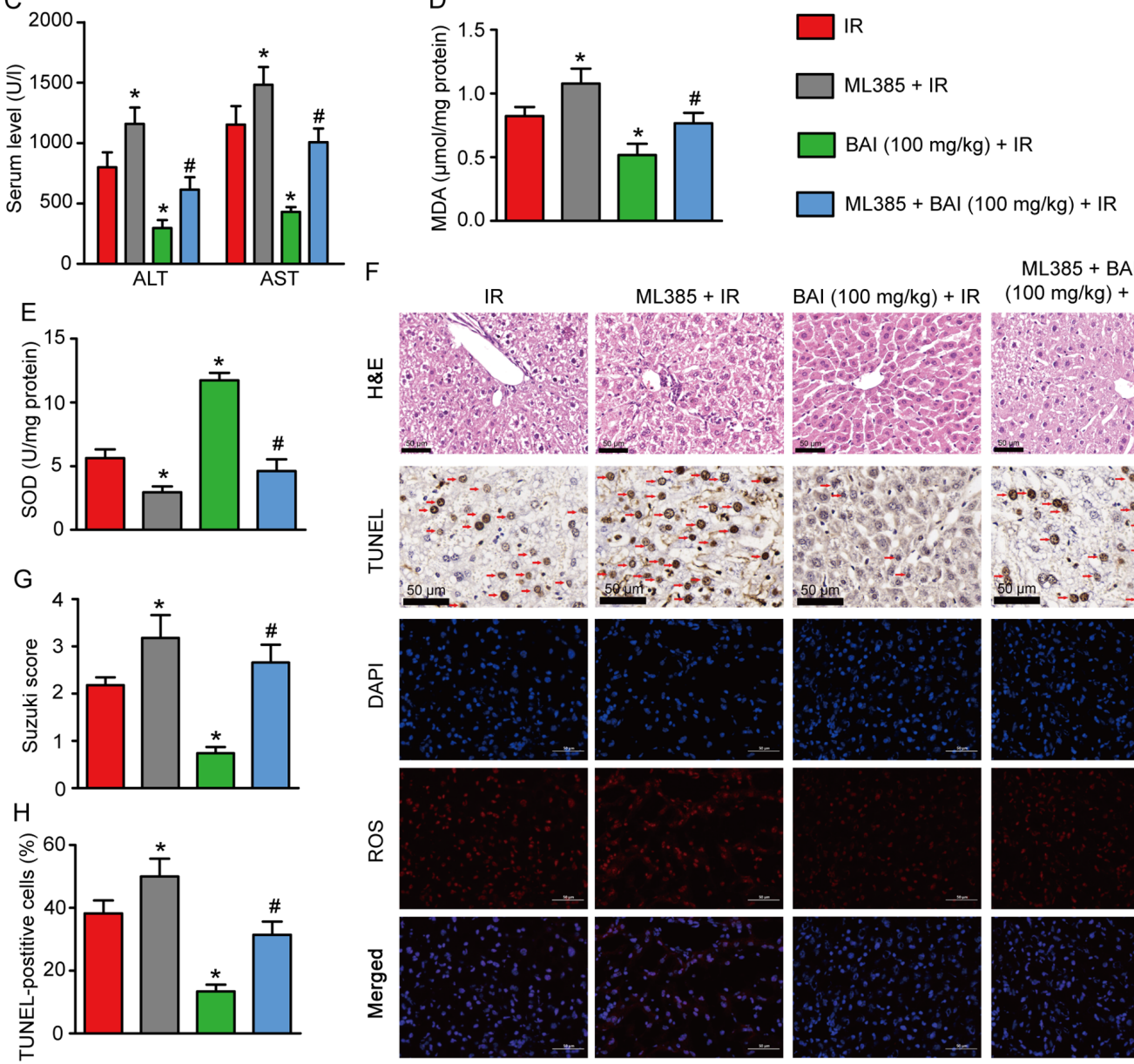
$\mathrm{BAI}(100 \mathrm{mg} / \mathrm{kg})+\mathrm{IR} \quad(100 \mathrm{mg} / \mathrm{kg})+\mathrm{IR}$

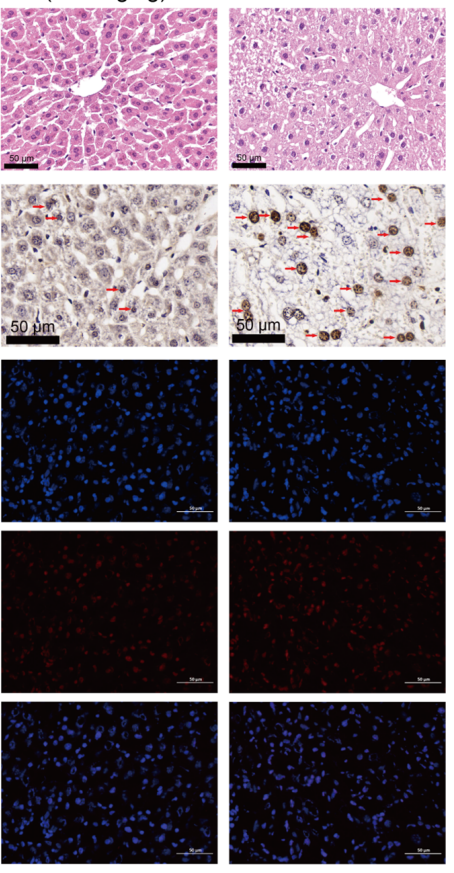

Figure 5. Nrf2/ARE pathway inhibitor reverse the protective effect of BAI on hepatic IR injury. Samples were collected $6 \mathrm{~h}$ after liver perfusion in all groups. (A) Levels of Nrf2 nucleoprotein, total HO-1, and NQO-1 protein were analyzed by western blotting. GAPDH and histone H3 were used as the internal controls. (B) Nrf2 nucleoprotein expression relative to histone H3 and HO-1 and NQO-1 protein expression relative to GAPDH. (C) Serum ALT and AST levels. (D) MDA content and (E) SOD activity were measured in liver tissue. (F) Pathological damage in the liver was measured by HE and TUNEL staining and ROS activity was measured by fluorescent-labeled DHE staining. Apoptotic cells are indicated by red arrows. Scale bar=50 $\mu \mathrm{m}$. (G) Suzuki score. (H) Percentage of TUNEL-positive hepatocytes. "P $<0.05$ vs. IR; ${ }^{\# P}<0.05$ vs. IR + BAI $100 \mathrm{mg} / \mathrm{kg}$. BAI, baicalein; IR, ischemia/reperfusion; Nrf2, nuclear factor E2-related factor 2; ARE, antioxidant response elements; HO01, heme oxygenase-1; NQO-1, NAD(P)H dehydrogenase quinone-1; MDA, malonaldehyde; SOD, superoxide dismutase; ALT, alanine aminotransferase; AST, aspartate aminotransferase; HE, hematoxylin and eosin; ROS, reactive oxygen species.

BAI upregulates Nrf2/ARE pathway protein expression levels in liver. Western blotting was used to detect the expression levels of Nrf2/ARE pathway-associated protein. Compared with the Sham group, the expression levels of Nrf2 nucleoprotein and total HO-1 and NQO-1 protein increased significantly in the IR and BAI $(100 \mathrm{mg} / \mathrm{kg}$ ) groups (Fig. 4A and B). Compared with the IR group, the expression levels of Nrf2 nucleoprotein and total HO-1 and NQO-1 protein in the BAI $(100 \mathrm{mg} / \mathrm{kg})+$ IR group were further increased following pre-treatment with BAI. However, there was no significant difference in the expression levels of Nrf2 nucleoprotein and total HO-1 and NQO-1 protein between the IR and BAI $(100 \mathrm{mg} / \mathrm{kg})$ groups.

Nrf2/ARE pathway inhibitor reverses the protective effect of BAI on HIRI. To confirm the role of the Nrf2/ARE pathway in BAI-treated mice following HIRI, the Nrf2 inhibitor ML385 was used. Compared with the IR group, the levels of Nrf2 nucleoprotein and total HO-1 and NQO-1 protein in the ML385 + IR group decreased significantly (Fig. 5A and B). Moreover, compared with 
the BAI $100 \mathrm{mg} / \mathrm{kg}+$ IR group, the expression of Nrf2 nucleoprotein and total HO-1 and NQO-1 protein in the ML385 + BAI $(100 \mathrm{mg} / \mathrm{kg})+$ IR group was significantly decreased. ML385 also aggravated liver injury (Fig. 5C, F and G), hepatocyte apoptosis (Fig. 5F and $\mathrm{H}$ ) and oxidative stress injury (Fig. 5D-F). These data suggested that the Nrf2/ARE pathway served a significant role in the protective effect of BAI against HIRI.

\section{Discussion}

BAI is the most abundant flavonoid in S. baicalensis and a key component of traditional Chinese medicine (31). Previous studies have shown that BAI attenuates HIRI via inhibition of the NF- $\kappa \mathrm{B}$ pathway and induction of autophagy $(22,23)$. Here, BAI pre-treatment significantly attenuated HIRI. BAI significantly decreased the elevated levels of AST and ALT and ameliorated the necrotic area of hepatocytes induced by HIRI. Additionally, pre-treatment with BAI inhibited oxidative damage and hepatocyte apoptosis. Pre-treatment with BAI promoted activation of the Nrf2/ARE pathway and alleviated liver injury during HIR, while Nrf2 inhibitor ML385 partially reversed the protective effect of BAI on HIRI. The present data indicated that BAI served a protective role in HIRI by regulating the Nrf2/ARE pathway to alleviate oxidative damage.

Multiple factors are involved in the occurrence and development of HIRI, such as oxygen free radical production, cell apoptosis, inflammatory reaction and calcium overload $(32,33)$. Oxidative stress induced by large amounts of ROS during reperfusion not only serves an important role in the early stage of hepatocyte injury but also participates in the later stage of inflammation (1). Excessive ROS beyond the clearance capacity of the liver damage lipids and proteins in cells, leading to cell death (34). The present results showed that the levels of ALT and AST in serum were increased following HIRI. Histological examination revealed that HIRI resulted in hepatocyte apoptosis, congestion and necrosis. This damage may be due to the formation of lipid peroxides by combination of excessive ROS and lipids in the cell membrane, resulting in destruction of the hepatocyte membrane $(35,36)$. The end product of lipid peroxides is MDA, which reflects the degree of cell peroxidation (37). SOD scavenges free radicals, which decreases oxidative damage caused by IR (38). BAI exhibits a well-known antioxidant capacity (39). Dong et al (10) found that BAI alleviates liver oxidative damage induced by high-level glucose. Pre-treatment with BAI increased the activity of SOD and decreased levels of ROS and MDA in liver tissue. Furthermore, pre-treatment with BAI significantly decreased the elevated levels of AST and ALT and ameliorated the necrotic area of hepatocytes induced by HIRI. These results showed that the protective effect of BAI against HIRI was mediated by alleviating oxidative damage to the liver.

The underlying mechanism of HIRI is complicated and not fully understood. Our previous study showed that [D-Ala2, D-Leu5]-Enkephalin significantly inhibits HIR-induced oxidative stress by activation of the Nrf2/HO-1 pathway (27). Numerous endogenous antioxidant protection genes, such as SOD (25) and phase II detoxification enzymes, such as $\mathrm{HO}-1$, are regulated by the Nrf2/ARE pathway (40). HO-1 and NQO-1 are two widely studied phase II enzymes regulated by Nrf2 that serve an important role in antioxidation $(38,41)$. Qin et al revealed that some hepatic metabolic enzyme genes of the Nrf2/ARE pathway, such as NQO1 and HO-1, were activated in BAI-treated hepatocytes (42). Shi et al also demonstrated that pre-treatment with BAI activates the Nrf2/ARE pathway to alleviate acetaminophen-induced oxidative damage (43). Kim et al (44) reported that baicalin alleviates liver IR injury by upregulating HO-1 expression. At the same time, baicalin serves an anti-inflammatory and antiapoptotic role by inhibiting activation of NF- $\mathrm{NB}$ and caspases 3 and 8 (44). Baicalin and BAI are primary flavonoids extracted from the dry roots of $S$. baicalensis (45). The molecular formula of BAI is $\mathrm{C}_{15} \mathrm{H}_{10} \mathrm{O}_{5}$, while that of baicalin is $\mathrm{C}_{21} \mathrm{H}_{18} \mathrm{O}_{11}$. The two molecules have similar pharmacological effects, such as anti-inflammatory, antioxidant and antiapoptotic effects. Qin et al (31) showed that BAI positively regulates the Nrf2/Kelch-like ECH-associated protein 1 (Keap1) pathway via Keap1-independent and -dependent pathways. In accordance with previous studies $(9,27)$, HIR lead to an increase in expression levels of $\mathrm{Nrf} 2$ nucleoprotein and total HO-1 and NQO-1 protein, which indicated that the antioxidant pathway was activated when the liver was subjected to IR. Moreover, pre-treatment with BAI further increased the expression levels of Nrf2 nucleoprotein and downstream antioxidative enzymes, such as SOD, HO-1 and NQO-1. These results indicated that pre-treatment with BAI promoted Nrf2 nuclear translocation and increased the antioxidant capacity of the liver. The protective role of BAI in HIRI was disrupted by the Nrf2 inhibitor ML385, which supported the key role of Nrf2/ARE in BAI-provided protection against HIRI.

Several S. baicalensis-derived mixtures or pure compounds have been approved as clinical therapeutic drugs in China; for example, BAI capsules are used to treat hepatitis (46). Hepatitis patients often develop liver cancer, and these patients with liver cancer and hepatitis often need extensive hepatectomy (47). HIRI is a common pathological process occurring in extensive hepatectomy (1). Therefore, it is worth studying whether BAI can be used before surgery in patients with hepatitis related liver cancer to decrease ischemia-reperfusion injury, as it is not only able to decrease ischemia-reperfusion injury, but also to decrease the adverse effect of hepatitis on the perioperative period. Nevertheless, there are certain limitations to the present study. The role of Keap1, an endogenous inhibitor of Nrf2, was not investigated. The dose selection of BAI was based on previous research (22). The maximum dose of BAI was $100 \mathrm{mg} / \mathrm{kg}$, which exhibited the most notable protective effect. Whether a higher BAI dose has a better protective effect requires further study. Based on the pharmacokinetics of BAI in vivo, the time and frequency of preoperative BAI should also be investigated in future.

The present findings showed that alleviation of oxidative stress by regulating the Nrf2/ARE pathway contributed to the protective effect of BAI against HIRI and BAI may be a promising therapeutic drug for the management of HIRI.

\section{Acknowledgements}

Not applicable.

\section{Funding}

The present study was supported by the National Natural Science Foundation of China (grant nos. 91949122 
and 81771674), the 111 Project (grant no. D17011), the Guangxi Key Research and Development Plan (grant no. 2018AD03001) and the Self-Funded Scientific Research Project of Guangxi Zhuang Autonomous Region Health Commission (grant no. Z20200575).

\section{Availability of data and materials}

The datasets used and/or analyzed during the current study are available from the corresponding author on reasonable request.

\section{Authors' contributions}

SH, GY and YZh designed the study. YZh, ZT, YZe, SC and $\mathrm{GH}$ performed the experiments. HH, YZe, JW, YZh, ZT and CQ analyzed the data. YZh and ZT wrote the original draft of the manuscript. SH and GY revised the manuscript. YZh and ZT confirm the authenticity of all the raw data. All authors read and approved the final manuscript.

\section{Ethics approval and consent to participate}

The present study was approved by the Animal Experimental Ethics Committee of the First Affiliated Hospital of Guangxi Medical University (approval no. 2017-KY-81771674-002). All the animals used were treated humanely.

\section{Patient consent for publication}

Not applicable.

\section{Competing interests}

The authors declare that they have no competing interests.

\section{References}

1. Zhai Y, Petrowsky H, Hong JC, Busuttil RW and Kupiec-Weglinski JW: Ischaemia-reperfusion injury in liver transplantation - from bench to bedside. Nat Rev Gastroenterol Hepatol 10: 79-89, 2013.

2. Busuttil R: Liver ischaemia and reperfusion injury. Br J Surg 94: 787-788, 2007.

3. Casillas-Ramírez A, Mosbah IB, Ramalho F, Roselló-Catafau J and Peralta C: Past and future approaches to ischemia-reperfusion lesion associated with liver transplantation. Life Sci 79: 1881-1894, 2006.

4. Elias-Miró M, Jiménez-Castro MB, Rodés J and Peralta C: Current knowledge on oxidative stress in hepatic ischemia/reperfusion. Free Radic Res 47: 555-568, 2013.

5. Jaeschke $\mathrm{H}$ and Woolbright BL: Current strategies to minimize hepatic ischemia-reperfusion injury by targeting reactive oxygen species. Transplant Rev (Orlando) 26: 103-114, 2012.

6. van Golen RF, van Gulik TM and Heger M: Mechanistic overview of reactive species-induced degradation of the endothelial glycocalyx during hepatic ischemia/reperfusion injury. Free Radic Biol Med 52: 1382-1402, 2012.

7. Hoek JB and Pastorino JG: Ethanol, oxidative stress, and cytokine-induced liver cell injury. Alcohol 27: 63-68, 2002.

8. Yi Z, Deng M, Scott MJ, Fu G, Loughran PA, Lei Z, Li S, Sun P, Yang C, Li W, et al: Immune-responsive gene 1/itaconate activates nuclear factor erythroid 2-related factor 2 in hepatocytes to protect against liver ischemia-reperfusion injury. Hepatology 72 : 1394-1411, 2020

9. Chi X, Zhang R, Shen N, Jin Y, Alina A, Yang S and Lin S: Sulforaphane reduces apoptosis and oncosis along with protecting liver injury-induced ischemic reperfusion by activating the Nrf2/ARE pathway. Hepatol Int 9: 321-329, 2015.
10. Dong Y, Xing Y, Sun J, Sun W, Xu Y and Quan C: Baicalein Alleviates Liver Oxidative Stress and Apoptosis Induced by High-Level Glucose through the Activation of the PERK/Nrf2 Signaling Pathway. Molecules 25: 599, 2020.

11. Liau P, Wu M and Lee C: Scutellaria baicalensisInhibitory Effects of Root Extract on Linoleic Acid Hydroperoxide-induced Lung Mitochondrial Lipid Peroxidation and Antioxidant Activities. Molecules 24: 2143, 2019.

12. Wang YS, Cho JG, Hwang ES, Yang JE, Gao W, Fang MZ, Zheng SD and Yi TH: Enhancement of Protective Effects of Radix Scutellariae on UVB-induced Photo Damage in Human HaCaT Keratinocytes. Appl Biochem Biotechnol 184: 1073-1093, 2018.

13. Lee W, Ku SK and Bae JS: Anti-inflammatory effects of Baicalin, Baicalein, and Wogonin in vitro and in vivo. Inflammation 38: 110-125, 2015.

14. Lu Y, Joerger R and Wu C: Study of the chemical composition and antimicrobial activities of ethanolic extracts from roots of Scutellaria baicalensis Georgi. J Agric Food Chem 59: 10934-10942, 2011.

15. Lin H, Hao Y, Wan X, He J and Tong Y: Baicalein inhibits cell development, metastasis and EMT and induces apoptosis by regulating ERK signaling pathway in osteosarcoma. J Recept Signal Transduct Res 40: 49-57, 2020.

16. de Oliveira MR, Nabavi SF, Habtemariam S, Erdogan Orhan I, Daglia $\mathrm{M}$ and Nabavi SM: The effects of baicalein and baicalin on mitochondrial function and dynamics: A review. Pharmacol Res 100: 296-308, 2015.

17. Wang H, Zhang Y, Bai R, Wang M and Du S: Baicalin Attenuates Alcoholic Liver Injury through Modulation of Hepatic Oxidative Stress, Inflammation and Sonic Hedgehog Pathway in Rats. Cell Physiol Biochem 39: 1129-1140, 2016.

18. Xi Y, Wu M, Li H, Dong S, Luo E, Gu M, Shen X, Jiang Y, Liu Y and Liu H: Baicalin Attenuates High Fat Diet-Induced Obesity and Liver Dysfunction: Dose-Response and Potential Role of

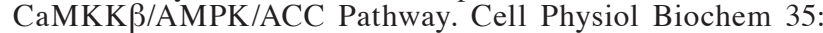
2349-2359, 2015

19. Xin HG, Zhang BB, Wu ZQ, Hang XF, Xu WS, Ni W, Zhang RQ and Miao XH: Treatment with baicalein attenuates methionine-choline deficient diet-induced non-alcoholic steatohepatitis in rats. Eur J Pharmacol 738: 310-318, 2014.

20. Sun H, Che QM, Zhao X and Pu XP: Antifibrotic effects of chronic baicalein administration in a $\mathrm{CCl} 4$ liver fibrosis model in rats. Eur J Pharmacol 631: 53-60, 2010.

21. Zhang Y, Shan L, Hua Y, Wang D, Zeng H, Liu R, Zhang W and $\mathrm{Hu} \mathrm{Z}$ : Baicalein selectively induces apoptosis in activated lymphocytes and ameliorates concanavalin a-induced hepatitis in mice. PLoS One 8: e69592, 2013.

22. Liu A, Huang L, Fan H, Fang H, Yang Y, Liu S, Hu J, Hu Q, Dirsch $\mathrm{O}$ and Dahmen U: Baicalein pretreatment protects against liver ischemia/reperfusion injury via inhibition of $N F-\kappa B$ pathway in mice. Int Immunopharmacol 24: 72-79, 2015.

23. Liu A, Huang L, Guo E, Li R, Yang J, Li A, Yang Y, Liu S, $\mathrm{Hu} \mathrm{J}$, Jiang $\mathrm{X}$, et al: Baicalein pretreatment reduces liver ischemia/reperfusion injury via induction of autophagy in rats. Sci Rep 6: 25042, 2016.

24. Ruiz S, Pergola PE, Zager RA and Vaziri ND: Targeting the transcription factor Nrf2 to ameliorate oxidative stress and inflammation in chronic kidney disease. Kidney Int 83: 1029-1041, 2013.

25. Wu P, Yan Y, Ma LL, Hou BY, He YY,Zhang L, Niu ZR, Song JK, Pang XC, Yang XY, et al: Effects of the Nrf2 Protein Modulator Salvianolic Acid A Alone or Combined with Metformin on Diabetes-associated Macrovascular and Renal Injury. J Biol Chem 291: 22288-22301, 2016.

26. Zhang H, Liu YY, Jiang Q, Li KR, Zhao YX, Cao C and Yao J: Salvianolic acid A protects RPE cells against oxidative stress through activation of $\mathrm{Nrf} 2 / \mathrm{HO}-1$ signaling. Free Radic Biol Med 69: 219-228, 2014.

27. Zhou Y, Zhang J, Lei B, Liang W, Gong J, Zhao C, Yu J, Li X, Tang B and Yuan S: DADLE improves hepatic ischemia/reperfusion injury in mice via activation of the Nrf2/HO-1 pathway. Mol Med Rep 16: 6214-6221, 2017.

28. Lu MC, Ji JA, Jiang ZY and You QD: The Keap1-Nrf2-ARE Pathway As a Potential Preventive and Therapeutic Target: An Update. Med Res Rev 36: 924-963, 2016.

29. Tsung A, Sahai R, Tanaka H, Nakao A, Fink MP, Lotze MT, Yang H, Li J, Tracey KJ, Geller DA, et al: The nuclear factor HMGB1 mediates hepatic injury after murine liver ischemia-reperfusion. J Exp Med 201: 1135-1143, 2005. 
30. Suzuki S, Toledo-Pereyra LH, Rodriguez FJ and Cejalvo D: Neutrophil infiltration as an important factor in liver ischemia and reperfusion injury. Modulating effects of FK506 and cyclosporine. Transplantation 55: 1265-1272, 1993.

31. Qin S, Deng F, Wu W, Jiang L, Yamashiro T, Yano S and Hou DX: Baicalein modulates Nrf2/Keap1 system in both Keap1-dependent and Keap1-independent mechanisms. Arch Biochem Biophys 559: 53-61, 2014.

32. Zhang Q, Lai Y, Deng J, Wang M, Wang Z, Wang M, Zhang Y, Yang X,Zhou X and Jiang H: Vagus Nerve Stimulation Attenuates Hepatic Ischemia/Reperfusion Injury via the Nrf2/HO-1 Pathway. Oxid Med Cell Longev 2019: 9549506, 2019.

33. Zhao Y, Cai H, Zhou P, Lin S, Pan Y and Liang X: Protective effect of ulinastatin on hepatic ischemia reperfusion injury through autophagy activation in Chang liver cells. J Cell Biochem 120: 14960-14970, 2019.

34. Reyes-Gordillo K, Shah R and Muriel P: Oxidative Stress and Inflammation in Hepatic Diseases: Current and Future Therapy. Oxid Med Cell Longev 2017: 3140673, 2017.

35. Konishi T and Lentsch AB: Hepatic Ischemia/Reperfusion: Mechanisms of Tissue Injury, Repair, and Regeneration. Gene Expr 17: 277-287, 2017

36. Ibrahim SG, El-Emam SZ, Mohamed EA and Abd Ellah MF: Dimethyl fumarate and curcumin attenuate hepatic ischemia/reperfusion injury via Nrf2/HO-1 activation and anti-inflammatory properties. Int Immunopharmacol 80: 106131, 2020.

37. Suji G and Sivakami S: Malondialdehyde, a lipid-derived aldehyde alters the reactivity of Cys34 and the esterase activity of serum albumin. Toxicol In Vitro 22: 618-624, 2008.

38. Liu D, Wang H, Zhang Y and Zhang Z: Protective Effects of Chlorogenic Acid on Cerebral Ischemia/Reperfusion Injury Rats by Regulating Oxidative Stress-Related Nrf2 Pathway. Drug Des Devel Ther 14: 51-60, 2020.

39. Gong WY, Zhao ZX, Liu BJ, Lu LW and Dong JC: Exploring the chemopreventive properties and perspectives of baicalin and its aglycone baicalein in solid tumors. Eur J Med Chem 126 844-852, 2017
40. Wu G, Zhu L, Yuan X, Chen H, Xiong R, Zhang S, Cheng H, Shen Y, An H, Li T, et al: Britanin Ameliorates Cerebral Ischemia-Reperfusion Injury by Inducing the Nrf2 Protective Pathway. Antioxid Redox Signal 27: 754-768, 2017.

41. Siegel D, Gustafson DL, Dehn DL, Han JY, Boonchoong P Berliner LJ and Ross D: NAD(P)H:quinone oxidoreductase 1: role as a superoxide scavenger. Mol Pharmacol 65: 1238-1247, 2004.

42. Qin S, Chen J, Tanigawa S and Hou DX: Gene expression profiling and pathway network analysis of hepatic metabolic enzymes targeted by baicalein. J Ethnopharmacol 140: 131-140, 2012.

43. Shi L, Hao Z, Zhang S, Wei M, Lu B, Wang Z and Ji L: Baicalein and baicalin alleviate acetaminophen-induced liver injury by activating Nrf2 antioxidative pathway: The involvement of ERK1/2 and PKC. Biochem Pharmacol 150: 9-23, 2018.

44. Kim SJ, Moon YJ and Lee SM: Protective effects of baicalin against ischemia/reperfusion injury in rat liver. J Nat Prod 73: 2003-2008, 2010.

45. Liang W, Huang X and Chen W: The Effects of Baicalin and Baicalein on Cerebral Ischemia: A Review. Aging Dis 8: 850-867, 2017.

46. Liu H, Ye F, Sun Q, Liang H, Li C, Li S, Lu R, Huang B, Tan W and Lai L: Scutellaria baicalensis extract and baicalein inhibit replication of SARS-CoV-2 and its 3C-like protease in vitro. J Enzyme Inhib Med Chem 36: 497-503, 2021.

47. Hwang S, Lee SG, Ahn CS, Kim KH, Moon DB, Ha TY, Song GW, Jung DH, Park JI, Ryu JH, et al: Prevention of hepatitis $\mathrm{B}$ recurrence after living donor liver transplantation: Primary high-dose hepatitis B immunoglobulin monotherapy and rescue antiviral therapy. Liver Transpl 14: 770-778, 2008.

c) (i) ( $)$ This work is licensed under a Creative Commons Attribution-NonCommercial-NoDerivatives 4.0 International (CC BY-NC-ND 4.0) License. 